\title{
Enhancing Skin Delivery of 5-Aminolevulinic Acid with Transferosome Using Lyso-Phospholipid and Surfactant
}

\author{
Insook Han†, Min Young Kang, Moonkyu Kim and Jung Chul Kim \\ Department of Immunology, School of Medicine, Kyungpook National University, Daegu 700-422, Korea
}

(Received January 7, $2011 \cdot$ Revised February 18, $2011 \cdot$ Accepted February 18, 2011)

\begin{abstract}
In order to enhance the clinical efficacy of 5-aminolevulinic acid-induced photodynamic therapy (ALAPDT), liposomal formulations using bulk hydrogenated phospholipids from soybean were introduced. Three types of lipids, S75-3, S100-3, and SL80-3 were used for formulating ALA. The $\mathrm{pH}$ of all the liposomal ALA is $4.5 \sim 5.5$ and the size is $50 \sim 200 \mathrm{~nm}$. All the liposomal formulations gave better ex vivo ALA skin penetration using nude mice skin in Franz cell than free ALA did. Among them, SL80-3 including 22\% of lyso-phosphocholine achieved excellent ALA penetration when compared with those of S75-3 and S100-3 which have only 1 2\% of lyso-phospholipids. S100-3 showed a little better results than S75-3 did. Addition of humectants (glycerine, propylene glycol, butylene glycol, betaine) in liposomal ALA formulated with SL80-3 produced little enhancing effect in ALA penetration. On the other hand, addition of surfactants (Tween 20,60 , Brij 72, 76, 78) in same liposomal system produced significant increase in ALA penetration. Among them, transferosomal system of lyso-phospholipid, SL80-3 and the surfactant, Brij76 showed the highest ALA penetration. Furthermore, this system also established the highest in vivo PpIX biosynthesis in hairy mice skin of C57BL/6. These results concluded that the transferosome of SL80-3 and Brij76 produced the best results in both ALA penetration and PpIX biosynthesis, and proved good correlation between them.
\end{abstract}

Key words - 5-Aminolevulinic acid (ALA), Skin penetration, Liposome, Transferosome, Lyso-phospholipid

Photodynamic therapy (PDT) using topical application of 5aminolevulinic acid has been widely applied for the treatment of neoplastic and non-neoplastic cutaneous diseases, including skin carcinoma, keratosis, psoriasis and acne vulgaris (Iinuma et al., 1994). When a precursor, ALA is administered to cells, an endogenous photosensitizer, protoporphyrin IX (PpIX) is synthesized and accumulated in vivo. Cells proliferating faster produce more PpIX than their slower growing ones, leading to an increased accumulation of PpIX in cells. When the light photoactivate PpIX, it is excited and transferred the energy to oxygen causing cell damaging and killing (Szeimies et al., 1996).

5-Aminolevulinic acid (ALA) is a highly acidic and hydrophilic compound and has a limitation on the penetration into the skin. In order to improve the penetration and reduce the toxicity of ALA, a number of liposome formulations have been investigated with or without the additive penetration enhancers (Tsai et al., 2002; Casas et al., 2006). Most of ALAcontaining liposomes using phospholipids and cholesterol promoted the ALA uptake and the PDT efficacy in comparision with that of free ALA. Liposome also improved the stability of

Corresponding Author:

Tel : +82-53-420-4879, E-mail : ishan@ $a$ knu.ac.kr

DOI : 10.4333/KPS.2011.41.1.019 drug or extracts for topical delivery (Noh et al., 2010), and their toxicity to cells and tissues (Han et al., 2005). Recently, nanosized liposomes received great interest in drug delivery system. Nanosized liposome smaller than $63.5 \mathrm{~nm}$ in diameter promoted the PDT efficacy, however, positively charged liposome showed no significant changes in PpIX accumulation and PDT efficacy (Kosobe et al., 2005). Ethanol and surfactants were also added into the liposomes (Fang et al., 2008). Average particle size of ethosome (10 15\% ethanol) and transferosome (10 50\% surfactant) were much less than that of liposome and were kept after long storage. In the result, the penetration ability of ALA with ethosome or transferosome was greater than that of the conventional liposome. Liposome using human stratum corneum (SC) lipids such as ceramides, cholesterols, free fatty acids and cholesteryl sulfate, showed higher skin retention of ALA on the epidermis without SC and dermis, with a decreasing of skin permeation compared to aqueous ALA solution. In this report, a distribution of vesicle size is $400-500 \mathrm{~nm}$ (Pierre et al., 2001).

In this work, we have formulated ALA with a variety of nanosized liposomes to increase the topical administration of ALA into skin. First experimental approach involves the examination of nanoliposomes formulated with typical phospholipids and lyso-phospholipids. Lyso-phospholipids increase significantly the fusogenicity of liposome to cells since it has 
only one phosphatidyl group within the molecules. Second approach is the application of the chemical enhancers. Polyols and surfactants were added to the liposomal solution, and the resulting transferosomes were evaluated in both ex vivo ALA penetration and in vivo PpIX biosynthesis, respectively.

\section{Materials and Methods}

\section{Chemicals and lipids}

5-Aminolevulinic acid hydrochloride was purchased from Fluka (Riedel-de Haën, Germany). Bulk hydrogenated phospholipids of S75-3, S100-3 and SL80-3 were obtained from Lipoid GmbH (Ludwigshafen, Germany; S, soybean source; $75,100,80$, percentage of phosphatidyl choline; -3 , maximum iodine value; L, lyso-phospholipids). S75-3 contains 70\% phosphocholine (PC), $2 \%$ lyso-PC, $10 \%$ phosphoethanolamine, $12 \%$ glyco-phospholipid and $6 \%$ other lipids. S100-3 contains $96 \%$ of (PC), $1.2 \%$ of lyso-PC and $2.8 \%$ of other lipids. SL80-3 contains $72 \%$ PC, 22\% lyso-PC and 6\% other lipids. Protoporphyrin IX, betaine, tween (TW) 20, TW 60, Brij 72, Brij76 and Brij 78 were purchased from Sigma (St. Louis, MO, USA).

\section{Preparation of liposome and transferosome}

First, microsized liposomal ALA was prepared by conventional rehydration method. Lipids were dissolved in chloroform:methanol=1:1 solution and the solvents was evaporated to produce the lipid film. Aqueous ALA in $0.5 \mathrm{M}$ hepes buffered saline ( $\mathrm{pH}$ 6.5) was then added and the whole mixture was shaked for $1 \mathrm{~h}$ at $250 \mathrm{rpm}$ and $40-50^{\circ} \mathrm{C}$. Additional polyols and surfactants were added before shaking. To make nanosized liposome, it was processed through the microfluidizer (Microfluidics M-110EH, Newton, MA, USA) at 15,000 psi x three times. Size of liposome was determined with a Nicomp submicron particle sizer (Model 370, Santa Barbara, CA, USA). The concentration of lipids was $50-100 \mathrm{mg} / \mathrm{mL}$ and that of ALA was $1 \sim 3 \% \mathrm{wt} / \mathrm{vol}$.

Percentage of encapsulation efficiency (\%EE) of ALA within liposome was determined using modified protamine aggregation method. In brief, liposomal ALA was mixed with an equal volume of protamine solution $(10 \mathrm{mg} / \mathrm{mL})$ and allowed to stand for $10 \mathrm{~min}$. Then, it was centrifuged at 2,000 $\mathrm{g}$ for $20 \mathrm{~min}$. Supernatant (S) was taken and pellet (P) was dissolved in methanol. \%EE was calculated as [(ALA in $\mathrm{P}) /(\mathrm{ALA}$ in $\mathrm{S}+\mathrm{P})] \times 100$. The amounts of ALA in S and $\mathrm{P}$ were determined by fluorimetric assay (Okayama et al., 1990). To prepare the fluorescent derivative of ALA, sample aliquots were mixed with dilute acetic acid $(20 \mathrm{~mL} / \mathrm{L})$, acetylacetone and formaldehyde solution $(100 \mathrm{~g} / \mathrm{L})$ at a volume ratio of $0.1: 2.5: 0.4: 1.0$. Whole mixtures were heated for $10 \mathrm{~min}$ at $100^{\circ} \mathrm{C}$ and then placed in ice-cold bath until analyzing. The fluorescence of ALA derivatives was measured by fluorescence spectrophotometer (LS55, PerkinElmer, UK) at 378/464 $\mathrm{nm}$ of excitation/emission wavelength.

\section{Ex vivo ALA penetration study}

After sacrificing of 5-week-old balb/c-nu mice (Hyochang Science, Korea), dorsal skin of full-thickness was excised and subsequently mounted in a Franz diffusion cell, kept at $37^{\circ} \mathrm{C}$. The donor cell contains $0.5 \mathrm{~mL}$ of either a formulated or an aqueous ALA solution and the acceptor cell includes isotonic phosphate buffered solution (PBS). Penetrated ALA was collected at regular intervals during 1 30 hours. To check the ALA retained in skin, tissues were cleaned with PBS and gently dried by pressing them with gauzes. Stratum corneum (SC) layers were removed by 5 tape strips using Scotch Crystal Tape ( $3 \mathrm{M}^{\mathrm{TM}}$, France) and used to extract ALA with PBS in SC. The remaining tissue was homogenized in PBS and filtered to check the ALA in epidermis and dermis. The amounts of ALAs in samples were determined by the same method described above and was expressed in $\mathrm{mg}$ or $\mathrm{ug} / \mathrm{g}$ of skin tissue.

\section{In vivo PpIX expression study}

Under general anesthesia, dorsal hairs 5-week-old C57BL/6 mice (Hyochang Science) were removed by electric shaving. After cleaning of shaved skin with warm water, $200 \mu \mathrm{L}$ of free or formulated ALA was topically applied using $1 \mathrm{~cm}^{2}$ gauze patch covered with Tegaderm (3M Health Care, MN, USA) for $30 \mathrm{~min}$ and post-incubated for $2 \mathrm{hrs}$ after removing patch. Treated skin was harvested and processed for $10 \mu \mathrm{m}$ frozen section using Cryostat-Microtome (Leica CM3050S, Germany). The fluorescent images of PpIX in slides were displayed via the Axioplan2 Imaging System (Carl Zeiss Vision, Germany). Under blue illumination (Ex. 365 nm, Em. 400 $\mathrm{nm}>$ ), the hair shafts and stratum corneum were observed in green while PpIX were observed in red. For the quantification of PpIX, the harvested skin samples were homogenized with liquid nitrogen and methanol, sonicated, and centrifuged to extract PpIX. The fluorescence of PpIX was determined by fluorescence spectrophotometer at $405 / 653 \mathrm{~nm}$ of excitation/ emission wavelength.

\section{Results}

S75-3, S100-3 and SL80-3 bulk lipids from Lipoid GmbH 


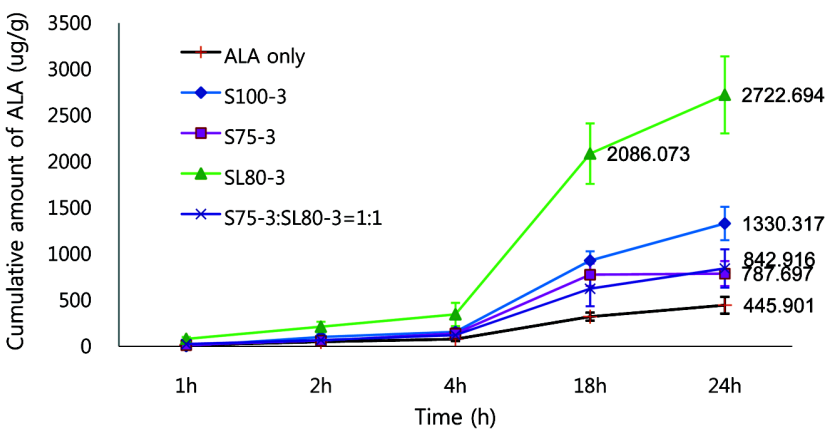

Figure 1. Penetration profiles of ALA through nude mouse skin from different liposomes (S100-3, S75-3, SL80-3 and S75-3:SL80$3=1: 1 \mathrm{w} / \mathrm{w}$ )

were employed to formulate the liposomal ALA and microfluidizer was used to make the nanosized liposome. The $\mathrm{pH}$ of $1 \sim 3 \%$ ALA aqueous solution was 2.0 2.5 that is highly acidic. In order to maintain the physical structure of the liposome, $0.5 \mathrm{M}$ hepes buffered saline of $\mathrm{pH} 6.5$ was used as a reconstitution solution to give the final liposomal ALA of $\mathrm{pH}$ 4.5 5.5. The average size of nanoliposome was 50 200 nm. The encapsulation efficiency of 1 3\% ALA within liposomes was $10 \sim 15 \%$ and used for experiment without purification of encapsulated ALAs. S75-3, S100-3 and SL80-3 includes 1.2, 2.0 and $22 \%$ of lyso-phosphocholine having only one fatty acid chain within molecule, while they contains 96, 92 and $72 \%$ of typical phospholipids having two fatty acids, respectively. In ex vivo skin penetraion results using nude mice skin (Figure 1), all the liposomal formulations showed better skin penetration than free ALA did. Significantly, liposomal ALA using SL80-3 (SL-ALA) showed much higher penetration than other formulations with S75-3, S100-3 and S75-3:SL80-3=1:1. They achieved similar profiles, however S100-3 showed a little better penetration at a later time. This result means that lyso-phosphocholine of SL80-3 (22\%) makes the liposome smaller and more fusogenic to penetrate into the skin easy and fast. However, no significant increase was shown in S75$3:$ SL $80-3=1: 1$ although $12 \%$ of lyso-phosphocholine was included in the formulation.

Different humectants of propylene glycol, butylene glycol, glycerin and betaine were added to liposomal solution of SLALA and the results were shown in Figure 2. All the polyols except butylene glycol contributed to enhance the skin penetration compared to free liposome. In other words, propylene glycol, glycerine and betaine produced similar penetration of ALA while butylene glycol did not improve it.

Different surfactants of TW20, TW60, Brij 72, Brij 76, Brij78 were also added into SL-ALA formulaion, and the resulting transferosomes were evaluated in ex vivo ALA pen-

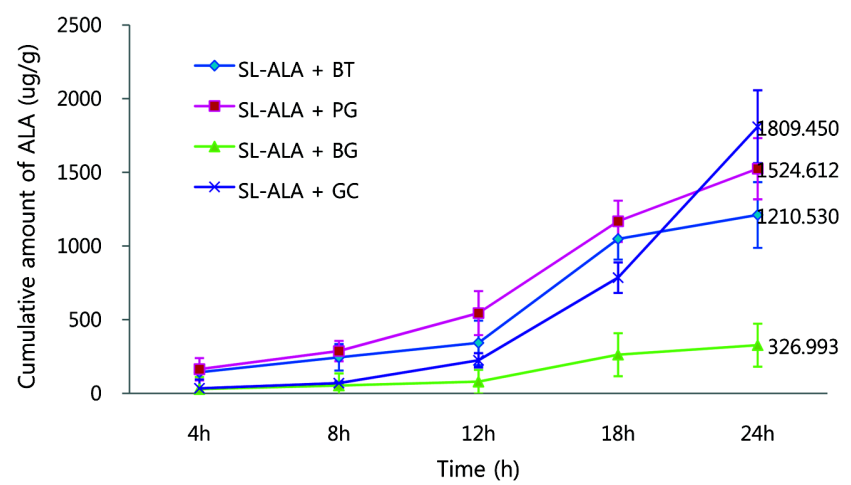

Figure 2. Penetration profiles of ALA formulated with SL80-3 (SL) and different humectants (BT, betaine; PG, propylene glycol; BG, butylene glycol; GC glycerin).

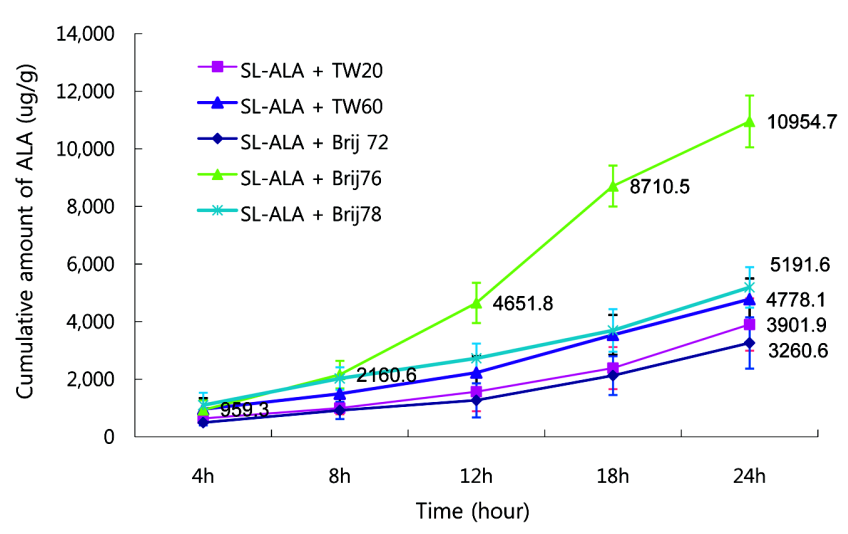

Figure 3. Penetration profiles of ALA formulated with SL80-3 (SL) and different surfactants (TW20, TW60, Brij72, Brij76, Brij78).

etration through the skin (Figure 3). Brij 76 improved the ALA penetration significantly and showed 2-3 times higher values at 12 24 hrs when compared with other surfactants. TW60 and Brij 78 showed similar enhancing effects (second group), while Brij72 and TW20 (third group) achieved similarly.

Finally, free ALA, liposomal ALA with SL80-3, and transferosomal ALA with SL80-3 and Brij76 were compared in both ALA penetration and retention in skin (Figure 4). Transferosomal ALA with SL80-3 and Brij76 (SL-ALA+Brij76) showed the best results in both ALA penetration and retention in skin without SC. ALA retention in SC showed no difference between formulations. SL-ALA containing propylene glycol (SL-ALA+PG) gave little improvement on the ALA penetration achieved with SL-ALA only. Similarly, transferosomal ALA including propylene glycol (SL-ALA+BRij76+PG) also rather decreased the ALA penetration established with the transferosomal ALA only.

Intensity of biosynthesized PpIX was finally evaluated after topical application of different formulations in hairy skin of C57BL6 mice (Figure 5). Again, transferosomal ALA for- 


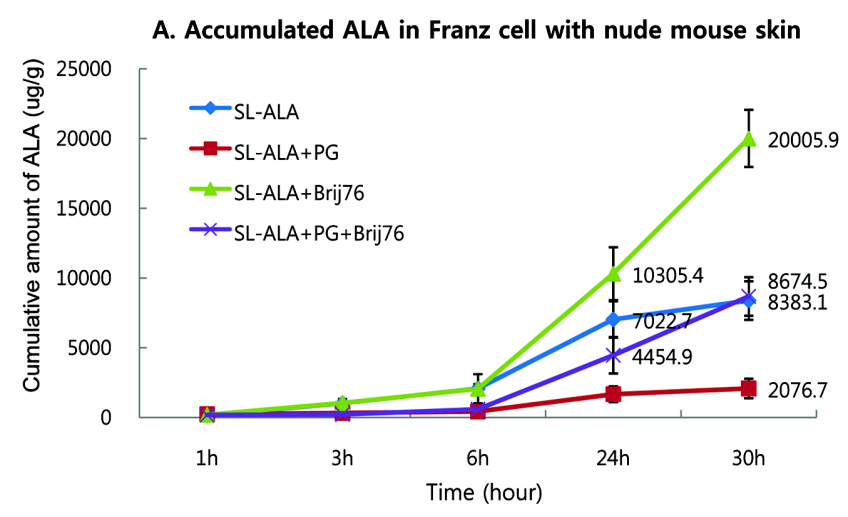

B. Retained ALA in nude mouse skin after $24 \mathrm{~h}$ delivery

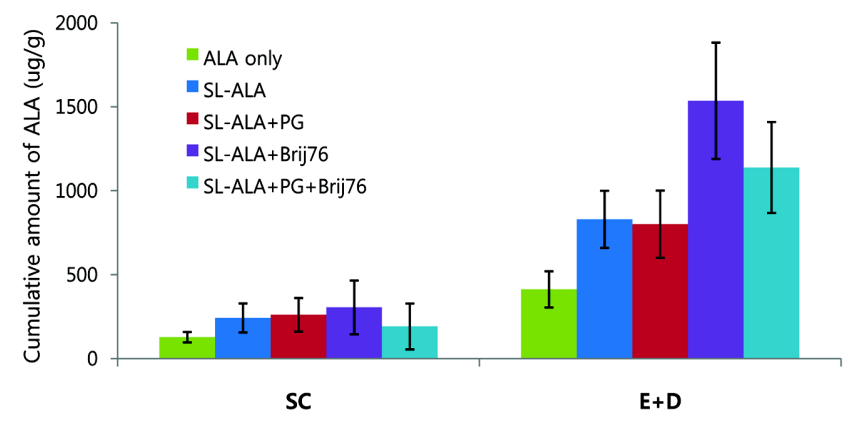

Figure 4. Ex vivo skin penetration and retention of ALA formulated with SL80-3 (SL) and different combination of additives (PG, polyethylene glycol. Brij76). mulated with SL80-3 and Brij76 showed the strongest intensity of PpIX in hair follicles and epidermis. No significant PpIX was found in dermis. The results of PpIX intensity in vivo corresponds well with those of ALA penetration ex vivo shown in Figure 4. Propylene glycol inhibited the PpIX intensity as well as the ALA penetration both in liposomal (SLALA) and transferosomal (SL-ALA+Brij76) formulation.

\section{Discussion}

Topical application or systemic administration of ALA was metabolized into PpIX which is majorly expressed in epidermis and pilosebaceous units (Divaris et al., 1990). Using this targeting, ALA-induced PDT have effectively treated epidermis- or pilosebaceous unit-related diseases. However, poor penetration of ALA within a biological environment limits the amounts of porphyrin accumulation enough to reach the photodynamic reaction in a limited time. Not only chemical enhancers (Malik et al., 1995; Casas et al., 2000) but also physical enhancers of iontophoresis (Rhodes et al., 1997; Lopez et al., 2003; Merclin et al., 2004) were introduced to increase topical delivery of ALA. Since ALA contains the cationic charge within the molecule, iontophoresis was very efficient using cathode application onto the ALAs. To date, many chemical a) Control

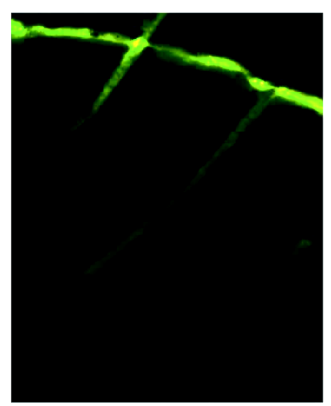

b) SL-ALA

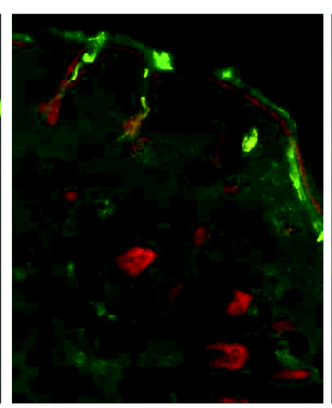

c) $S L-A L A+P G$

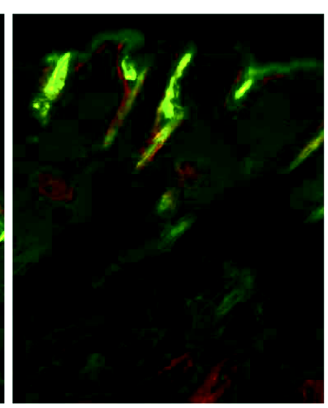

d) SL-ALA+Brij76

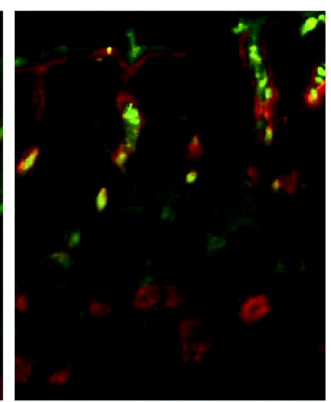

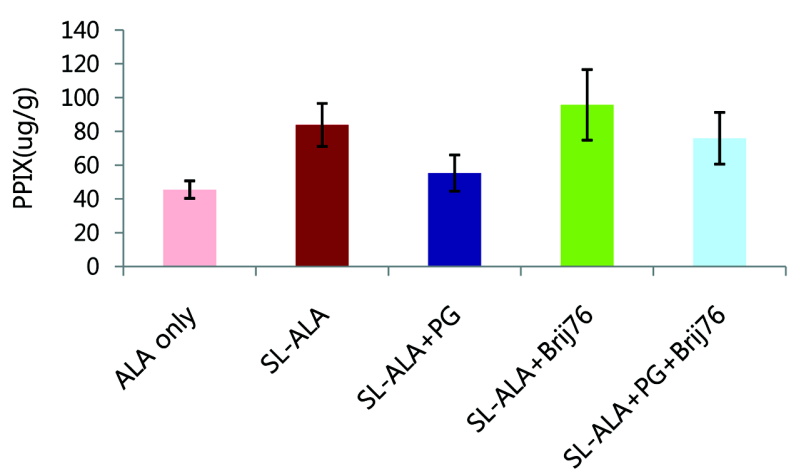

Figure 5. In vivo PpIX expression induced by different formulations of ALA in C57BL6 mice skin after 30 min topical application and $2 \mathrm{~h}$ postincubation. Control, 14 days-old C57BL/6 mouse dorsal skin; b) ALA only, 3\% ALA at 200mM HEPES solution (pH=5.5); c) SLALA, liposomal ALA formulated with SL80-3; d) SL-ALA+Brij76, transferosomal ALA formulated with SL80-3 and Brij76. 
vehicles have also been used to deliver ALA including emulsions, liposomes, nanoparticles, and a lipid sponge form (Merclin et al., 2004). Liposome is generally accepted in various delivery strategy, which enhance the capillary permeation of hydrophilic/lipophilic drugs and localize them to target tissues. It is also non-toxic and biodegradable. Nanosized liposome showed better delivery of ALA than the typical microsized liposome (Casas et al., 2006; Venosa et al., 2008). In addition, previous studies demonstrated that phospholipids for liposome can exhibit their enhancing effect on the skin in the presence of organic solvent such as ethanol (Fang et al., 2008) and small polyols of glycerin, propylene glycol or tetraglycol. Knowledge of the relationship between ALA delivery and the effect of formulation is important for designing optimal formulations and treatment schedules for topical ALA-PDT (Tsai et al., 2002; Leeuw et al., 2010).

Based on these previous reports, we have formulated nanosized liposome including lyso-phospholipids to enhance the delivery and fusogenicity of ALA into cells and tissues. All the formulations showed better delivery and more stability than free ALA did. Nanosized liposomal ALA produced also stronger PpIX intensity than microsized one. Particularly, SL80-3 achieved excellent penetration of ALA into the mouse skin. Since SL80-3 contains $22 \%$ of lyso-phospholipids which have high cell-fusogenicity and form micelle structure by itself, the liposome formulated with SL80-3 seems to be more like transferosome system rather than the conventional bi-layered liposome structure. On the other hand, S75-3 and S100-3 includes only $1 \sim 2 \%$ of lyso-phospholipids and their original structure of bi-layered liposomes were not changed. Furthermore, transferosome formulated with SL80-3 and the surfactant, Brij76 showed more significant increases in ex vivo ALA penetration in Franz cell and in vivo PpIX biosynthesis in skin tissue. Lyso-phospholipids with surfactant accelerated the production of micelle structure with high fusogenicity and the disruption of stratum corneum increasing the skin penetration of ALA. In the result, the accumulation of biosynthesized PpIX was increased. However, polyols did not improve the topical penetration of ALA. Decrease by adding of polyols may be caused by the increase the viscosity, which retards the delivery.

In conclusion, transferosome system established by the addition of lyso-phospholipids and/or surfactant into conventional phospholipids produced excellent skin penetration of ALA and high accumulation of PpIX. Furthermore, nanosized transferosome exhibited better ALA penetration and faster PpIX biosynthesis than its liposome. Controlling the ratio of lyso-lipids and surfactants would be next subject for designing optimal transferosomal formulations for the success of topical ALA-PDT.

\section{Acknowledgements}

This work was supported by the Grant of the Korean Ministry of Education, Science and Technology (The Regional Core Research Program/Anti-aging and Well-being Research Center).

\section{References}

Casas A., Batlle, A., 2006. Aminolevulinic acid derivatives and liposome delivery as strategies for improving 5-aminolevulinic acid-mediated photodynamic therapy, Curr. Med. Chem., 13, 1157-1168.

Casas, A., Fukuda, H., Venosa, G., Del, A.M., Batlle, C., 2000. The influence of the vehicle on the synthesis of porphyrins after topical application of 5-aminolaevulinic acid. Implications in cutaneous photodynamic sensitization, Br. J. Dermatol., 143, 564-572.

Divaris, D.X.G., Kennedy, J.C., Poittier, R.H., 1990. Phototoxic damage to sebaceous glands and hair follicles of mice after systemic administration of 5-aminolaevulinic acid correlates with localized protoporphyrin IX fluorescence, Am. J. Pathol., 136, 891-897.

Fang, Y.-P., Tsai, Y.-H., Wu, P.-C., Huang, Y.-B., 2008. Comparision of 5-aminolevulinic acid-encapsulated liposome versus ethosome for skin delivery for photodynamic therapy, Int. J. Pharm., 365, 144-152.

Han, I., Jun, M.S., Kim, S.K., Kim M., Kim J.C., 2005 Expression pattern and intensity of protoporphyrin IX induced by liposomal 5-aminolevulinic acid in rat pilosebaceous unit throughout hair cycle, Arch. Dermatol. Res., 297, 210-217.

Iinuma, S., Farshi, S.S., Ortel, B., Hasan, T., 1994. A mechanistic study of cellular photodestruction with 5-Aminolevulinic acidinduced porphyrin, Br. J. Cancer, 70, 21-28.

Kosobe, T., Moriyama E., Tokuoka, Y., 2005. Size and surface charge effect of 5-aminolevulinic acid-containing liposomes on photodynamic therapy for cultivated cancer cells, Drug Dev. Ind. Pharm., 31, 623-629.

Leeuw, J., Beek, N., Bjerring, P., Neumann, H.A., 2010. Photodynamic therapy of acne vulgaris using 5-aminolevulinic acid $0.5 \%$ liposomal spray and intense pulsed light in combination with topical keratolytic agents, J. Eur. Acad. Dermatol. Venereol., 24, 460-469.

Lopez, R., Bentley, M., Delgado-Charro, M., Guy, R., 2003. Optimization of aminolevulinic acid delivery by iontophoresis, $\mathrm{J}$. Con. Rel., 88, 65-70.

Malik, Z., Kostenich, G., Roitman, L., Ehrenberg, B., Orenstein, A., 1995. Topical application of 5-aminolaevulinic acid, DMSO and EDTA: protoporphyrin IX accumulation in skin and tumours of mice, J. Photochem. Photobiol. B., 28, 213218.

Merclin, N., Bender, J., Sparr, E., Guy, R.H., Ehresson, H., Engstrom, S., 2004. Transdermal delivery from a lipid sponge 
phase- iontophoretic and passive transport in vitro of 5-aminolevulinic acid and its methyl ester, J. Con. Rel., 100, 191198.

Noh, S.M., Park, D.-E., Im, S., Kim. S., Kim, Y.B., Oh, Y.-K., 2010. The effects of storage conditions on the stability of porcine placenta extract-loaded liposome formulations, J. Pharm. Invest., 40(3), 187-192.

Okayama, A., Fujii, S., Miura, R., 1990. Optimized fluorometric determination of urinary delta-aminolevulinic acid by using pre-column derivatization, and identification of the derivative, Clin. Chem., 36, 1494-1497.

Pierre, M.B., Tedesco, A.C., Marchetti, J.M., Bentley, M.V., 2001. Stratum corneum lipids liposomes for the topical delivery of 5aminolaevulinic acid in photodynamic therapy of skin cancer: preparation and in vitro permeation study, BMC Dermatol., 1, 5-10.
Rhodes, L.E., Tsoukas, M.M., Anderson, R.R., Kollias, N., 1997. Iontophoretic delivery of ALA provides a quantitative model for ALA pharmacokinetics and PpIX phototoxicity in human skin, J. Invest. Dermatol., 108, 87-91.

Szeimies, R.M., Calzavara-Pinton, P., Karrer, S., Ortel, B., Landthaler, M., 1996. Topical photodynamic therapy in dermatology, J. Photochem. Photobiol. B., 36, 213-219.

Tsai, J.-C., Chen, I.-H., Wong, T.-W., Lo, Y.-L., 2002. In vitro/in vivo correlations between transdermal delivery of 5-aminolevulinic acid and cutaneous protoporphyrin IX accumulation and effect of formulation, Br. J. Dermatol., 146, 853-862.

Venosa, G.D., Hermida, L., Batlle, A., Fukuda, H., Defain, M.V., Mamone, L., Rodriguez, L., Macrobert, A., Casas, A., 2008. Characterisation of liposomes containing aminolevulinic acid and derived esters, J. Photochem. Photobiol. B., 92, 1-9. 